\title{
Scenario building for E-Government in 2020: Consolidating the results from regional workshops
}

\author{
Marijn Janssen, Patrick van der Duin, René \\ W. Wagenaar \\ School of Technology, Policy and \\ Management \\ Delft University of Technology \\ Jaffalaan 5, NL-2600 GA, Delft \\ The Netherlands \\ Tel. +31152781140 \\ E-mail: \{m.f.w.h.a.janssen, p.vanduin, \\ r.w.wagenaar tudelft.nl \\ Sharon Dawes \\ Center for Technology in Government \\ University at Albany \\ 1535 Western Avenue \\ Albany, NY 12203-3513 \\ Tel: (518) 442-3892 \\ Fax: (518) 442-3886 \\ E-mail:sdawes@ctg.albany.edu
}

\author{
Melanie Bicking, Maria A. Wimmer \\ University of Koblenz-Landau \\ Institute for IS Research, Research Group \\ eGovernment \\ Universitaetsstr. 1, 56070 Koblenz \\ Germany \\ Tel: +492612872646 \\ Fax: +492612871002646 \\ E-mail: \{bicking,wimmer\}@uni-koblenz.de
}

\begin{abstract}
E-government research is currently at a stage of consolidation and new orientation. Smaller steps of government modernization have in part been successfully implemented; larger ones still lie ahead of us. Within an EC funded project, a roadmap for egovernment is being defined. Thereby, scenario building about the future is being used to grasp pictures of the future. From these future scenarios, research actions shall be derived.

In this paper we present the results of the scenario building workshops in four regions for e-government in the year 2020. In total 15 scenarios were developed shaping aspects of different dimensions of alternative futures. A wide range of topics were addressed, varying from interoperability, division of power, corruption, cyber war to changing democratic systems, religious activities, participations and the role of individualisms in society. Next steps are to assess the scenario results in respect to the current EC agenda and to derive from there actions of research in order to ensure that desired futures will come true and that unwanted futures are being avoided.
\end{abstract}

\section{Introduction}

Today, governments everywhere in the world face new pressures associated with becoming an information society. Public agencies are more often asked to interlink and collaborate in different fields. Consequently, they need a holistic and integral view that integrates technology, processes, organization and humans [18].

Most current planning activities investigate only the short-term future and the question remains how egovernment will look like in 2020. How will governments evolve beyond the next five years - e.g. till 2020? What kind of activities will the public sector be responsible for in 2020, and how will it carry out the work? What kind of technology will be in use in 14 years from now and which values will become important by that time? The future of e-government is difficult or even impossible to predict, as egovernment is complex and multifaceted. Many existing studies describe visions of the future until 2010 and rely on trend analysis [1]. In contrast, in this paper we will look at a longer time horizon of 2020 that cannot rely on trend analysis alone. 
Scenario building has become an integral part of the innovation and policy-making process [6]. The goal of the scenario-method is to stimulate different perspectives or images of the future of a certain topic in order to develop a better view on possible future directions. Scenarios are archetypal images of the future that nevertheless often interpret current realities. Scenarios are internally consistent, mutually different and plausible stories about a path from now into the future. Scenarios can include contrasting perspectives on the future and can support better policy development.

In this paper we present the findings of four regional scenario building workshops that are part of a research program supported by the European Commission to develop a research agenda for moving toward e-government in 2020. We compare the findings to derive overlapping topics and variations in dimensions that may influence e-government in 2020. Topics of the scenarios represent key areas to be addressed in future research. With the assessment of impact and (un)certainty if a direction within a topic will come true, the gathered topics and scenarios represent an instrument for policy-makers to help them make strategic decisions on actions to take to ensure desired futures materialize and to avoid undesired ones.

In the following section we present the background of scenario building. Thereafter the research approach is presented, and the four regional scenario building workshops are summarized. The aspects expected to have a large influence on e-government in 2020 are aggregated and discussed in section five. Finally conclusions are drawn and our future research activities are presented.

\section{Scenario building background}

In future research, scenario building is a method to stimulate different perspectives or images of the future of a certain area to gain more insight into possible future developments [10]. Based on the insights from such visionary views, concerted and focused actions can be derived to positively impact future developments.

Scenario building strongly differs from forecasting. While forecasting predicts the near future based on the extrapolation of past and current developments, scenario building cuts off the past and requires us to look solely into the future. Of course, scenarios have some historical roots. Every expert reflects on his or her past and current knowledge of actual developments and from thereof extrapolates his or her view on potential futures. Figure 1 (based on [2]) shows that certain developments will continue for some time into the future (the single dashed line). In this period reliable forecasts can be made. However, after a certain length of time it becomes impossible to extend the line (or development) because there is too much uncertainty. Forecasting with high accuracy is then almost impossible. From this point onwards, scenarios (the four diverging dotted lines) can be used to explore the future. The moment at which there is too much uncertainty to extrapolate may differ for different variables. For example, forecasts concerning the birth rate are usually relatively accurate for a time horizon of 15 years, whereas correct predictions of the number of mobile telephones are aimed at only two years into the future.

The use of scenarios within both commercial and non-commercial organizations received a significant boost in the 1970s when Shell was able to anticipate the oil-crisis in good times for the oil-industry by using scenarios [17]. Together with the Rand Corporation they turned simple 'what if' exercises performed by national armies into a fully-fledged futures research method [4][9][15]. There are many different methods of scenario development (e.g. [2],[9],[16]) Creativity and the involvement of subjective opinions by (possibly biased) humans are important ingredients of all scenario-methods [6].

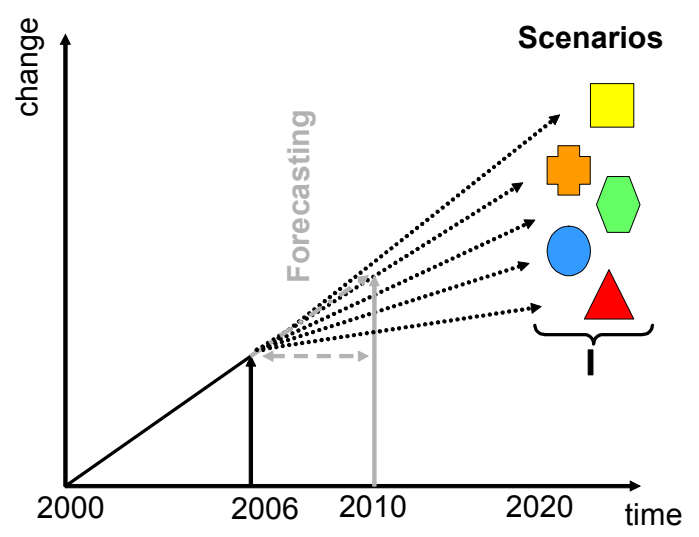

Figure 1: Scenario development (based on [2])

Scenario building does not pretend to predict the future. If an adequate environment is being provided, the technique facilitates the identification of essential aspects of an unpredictable future. When applying the scenario building methodology, one must be aware that the implicit biases of participating agents can never be turned off fully. However, it is important that during scenario workshops the activities actively avoid 
dwelling on past and current states that could then be just projected to the future as is done in the forecasting.

To develop scenarios in a conducive environment, it is of crucial importance that moderators should make clear that scenarios are not predictions; they are not merely extrapolations from current and past evolution [6]. They should also make clear that scenarios are neither good nor bad. Participants should be motivated to think the unthinkable [6],[11]. During a scenario workshop, reaching a consensus is not necessary [6]. Scenarios may differ: even extreme opposite scenarios can - and should - be developed. In the case of extremely opposing ideas, scenario axes should be identified to bring the extremes into relation. To do this, moderators need to make sure that participants' do not criticism is exerted on each other's ideas.

Scenario building focuses on a specific topic. To ensure that the essentials of a scenario description are captured, a workshop program, approach and scenario template needs to be provided. In the next section we use this information to present our scenario building approach, and discuss the workshop program and the template for describing the scenarios.

\section{Research methodology}

\subsection{Overall research approach}

The objective of the scenario building is to draw plausible and internally consistent scenarios of how the future will look in 2020. In the first phase the scenario-building methodology as described in this paper was determined. In the second phase 3 European workshops and one US workshop was conducted resulting in 15 scenarios and workshop reports were written. As this is part of an EU funded project, the focus of the workshops was on Europe. The US workshop was conducted to include a non-European perspective, which should contribute to the diversity.

In the third phase, the results of the four workshops were analyzed. From each scenario the main topics of interest were derived. The idea is that dimensions within each topic of interest can result in alternative futures. An example from the workshop can demonstrate this approach. The topic privacy might contain, as a dimension that privacy is offered at the expense of security in 2020 vs. privacy will not be scarified as terrorism is less an issue. Another dimension of the privacy topic is that privacy will be better understood and therefore it could be better protected, new content-dependent mechanism are able ensure privacy vs. privacy is not understood and cannot be ensured using technology. For all scenarios all topics were analyzed and the occurrence in each scenario was counted. Although counting occurrences provides no statistical evidence for generalization, it gives us an indication in how many regions might be affected by alternative futures. The collection of all topics and dimensions determines possible futures.

\subsection{Workshop design}

The workshops began with presentations of the background and scenario building approach. Thereafter the participants were split up in smaller groups of three to six participants. Each subgroup was asked to develop one scenario for e-government in 2020. A moderator helped each group in getting started with the visionary discussions, facilitated the process.

All participants then came together to present their scenarios and to discuss them with the others. After the scenario discussion in the plenum, a final round of identifying and categorizing scenario dimensions was carried out. In this activity the dimensions were assessed for both impact and likelihood.

Four regional workshops were conducted in different parts of the world and all followed the same approach during the period from April-June 2006. The main reason for conducting a number of regional workshops is to capture the diversity in regional differences. As this research is aimed at the European level, three workshops were conducted in Europe, covering the Eastern, Northern and Central European regions and one workshop outside Europe was conducted to ensure that visions outside the European domain were taken into account.

\subsection{Contextual and interaction environment}

To get valuable images of the future, it was crucial to base on a holistic framework that would ensure that the experts imagined all relevant aspects of their scenarios that interdependently impact each other in their interplay in the context of e-government. Figure 2 shows the framework guiding the scenario development. The following four aspects are the core of this framework (cf. inner circle):

- Society, environment and culture

- Governments and Administration,

- ICT developments and innovation

- Economics, efficiency and effectiveness.

When deriving scenario descriptions, moderators had to secure that each of the aspects was described in 
the scenario(s) developed. Apart from the single aspects, the relations (arrows) were most interesting: how will governments use ICT to serve citizens (society) and what economic values will be important thereby? This is just one of many questions, moderators posed to the experts in the group discussions during scenario development.

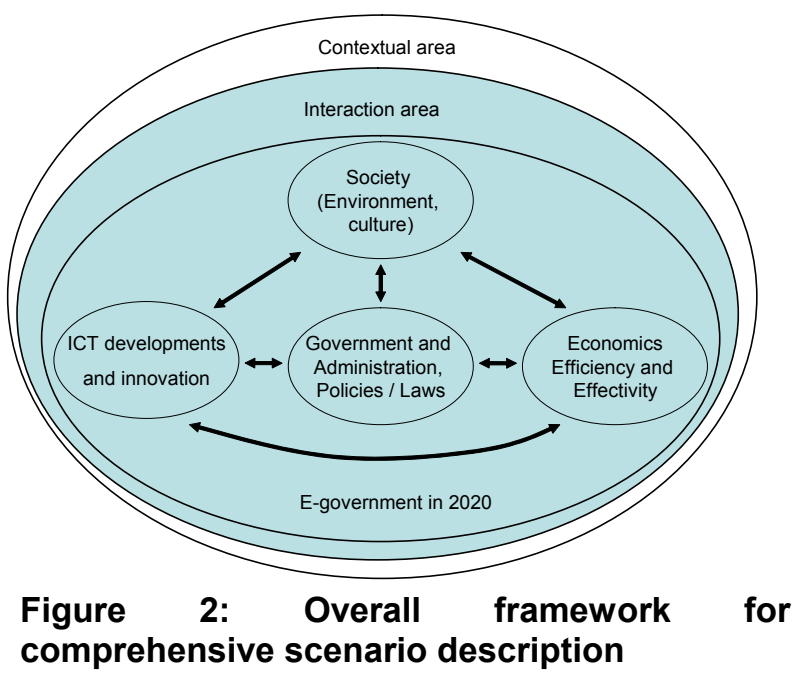

Certainly, the single aspects of e-government and their interplay need to be reflected by their surrounding interaction environment and their global context (e.g. [8][10]). In this model the central scenario topic, in our case e-government in 2020, is surrounded by two areas, the interaction area and the contextual area.

The interaction environment contains actors such as citizens, business, other governmental agencies, NGOs, PPP, suppliers, customers, politicians, legislators, and other stakeholders in the society. Also the type of services provided and the various modes of participation and technology used to involve people in policy-making processes are part of this environment. Their influence on the scenario-subject is direct and takes place within a short time-horizon. Due to the dependencies, it is likely that the actors influence the scenarios.

The contextual environment is further away from egovernment. It contains social, technological, economic, political and environmental factors by which we mean subjective forces such as basic values, societal trends and developments. Examples of such forces or trends could be the speed of technological development, individualism in society, changing political climate, economic development, migration patterns or constitutional values like privacy and human rights. These factors influence the scenarios in the long term but often are not affected by egovernment developments. For example, it is not possible for actors involved with E-government to influence the birth rate.

\section{Scenario development workshops results}

A total of 15 different scenarios were developed in 4 regional workshops. In the following subsections we provide a short description of the scenario building workshop and a summary of the scenarios developed.

\subsection{Eastern-Europe workshop}

The first workshop of the regional workshops was held in Prague during the Eastern European E-Gov days (http://www.ocg.at/egov/eeegovdays06.html). In total 14 people representing 6 European countries and mainly coming from the academic world attended the workshop. During the workshop the group was split up into four subgroups creating four scenarios. The scenarios can be summarizes as follows.

1. The ambient government of Europe. In 2020 society will have changed and grown older. Government will be ambient, providing basic services for all, but the private sector is expected to gain more power by delivering extended services for those who can afford them. The democratic system will have eroded and there is a large divide between the haves and haves not.

2. Sustainable and pervasive governments. Government services and participation in policymaking and law enforcement (whatever) is accessible whenever/ wherever and will be ambient. The public sector delivers services in all areas. Governments participate in community to ensure close relationships with its citizens. The EU has grown strong and social systems have grown even stronger. A strong education system is the major factor to ensure nations' competitive advantage

3. Government as industry puppet. Government is fragmented and performs a limited number of functions. Almost all service provisioning is left to the private sector and industry rules the world and influences policy-making to a large extent. The EU is fragmented and weak. Privacy is sacrificed for business purposes and businesses have a large influence on politicians and their decisions.

4. Orchestrating government. Government is primarily aimed at understanding the needs and wishes of their constituencies and on coordinating the 
fulfillment of their needs. There is a small and lean public administration aimed at directing the implementation, execution and enforcement of policymaking. Most functions are performance by the private sector within the policies and conditions set by governments. There is an emphasis on collecting information, processing to improve policy-making.

\subsection{Northern-Europe workshop}

In Vilnius a workshop was held with 18 people. A number of consultants and academia and 11 representatives of ministries and other governmental organizations participated in the workshop. Due to its diversity the group was split up into three subgroups, which resulted in three main scenarios as summarized below.

1. Human centred government. In 2020 the EU member states will grow closer together. EU-wide seamless data exchange will occur. Governments will provide user centric services in a fully automatic fashion. Individuals will belong to multiple, global and diverse communities of interest. People are globetrotters and continue to learn from anywhere. Privacy will be better understood and therefore it could be better protected. Participation is not only possible through voting but via the exchange of opinions and feedback. There are technologies to direct and manage the information overload.

2. The new e-world order. The EU is extended to the east. Seamless trading with Russia as part of an extended Schengen agreement. Virtual territories make up borders and voting rights. The world is divided between democratic and non-democratic countries. Privacy is made content-dependent. Government innovation is mainly coming from business, government focuses on providing standard services. We see the end of party politics, and participation has both a central and local focus, with the rise of many horizontal, virtual, communities. Interoperability between EU countries and between EU, central and local levels are accomplished

3. Collaborative and highly networked society. The hierarchical system of the society is flatter and communities of interests are used to support participation in policy-making. The biggest problem will be possibilities for total surveillance and the "black technologies". Partnerships between different interest groups will be organised via networks. Several serious problems will arise as well (for example, social divide - only the "in-crowd" will have possibilities to participate in serious decisions leaving the grassroots only simple and irrelevant ones.)

\subsection{Central-Europe workshop}

In the Central-Europe workshops, in total 19 persons participated coming from governments, software providers and consultancy organizations. The session resulted in the following scenarios.

1. A brave new world. In 2020 the populations' exigency of more security in Europe leads to a brave new world in which government deploys more and more ICT supported control and monitoring mechanism in order to satisfy this exigency. Citizens and business are strongly regulated and values like privacy protection are subordinated to the public welfare.

2. Cooperative state. In 2020 all federal systems have a central decision making policy whereby federal and state institutions cooperate very closely. Public and private sector have developed many cooperative and collaborative structures. The public sector is responsible to ensure public service provision but the private sector implements these services. From this it follows that as little personal data as possible shall be requested and stored, as well as data specification and storage shall not be controlled by businesses nor by governments.

3. Liberal night watcher state. Governments are restricted to provide and supply the minimum of public services necessary. Individualism and personal responsibility are the most important values of society. Government becomes a small institution and the private sector performs most of its functions.

4. Divergence and parallelism. In 2020 particular interests are more important than the general public welfare. Nationalization and individualization split Europe into its single member states. Security, in particular social-security-awareness, is a central topic. Member states monitor their citizens and collect and connect all relevant information about them for knowledge generation, decision making and invention actions Strongly competition between the member states takes place. Citizens are not willing to accept 'bad' technologies. Businesses have a high impact on government and society.

\subsection{US workshop}

The workshop in the USA was associated with International Digital Government Research Conference in San Diego (http://dgrc.org/dgo2006/). It included 27 participants representing 10 countries, four of them outside Europe. Most participants were academics, with a few private sector and government managers. The following scenarios were developed. 
1. Demographics Rule: In 2020, older people will command an increasingly large proportion of public services, especially health care. This generation will also control a huge proportion of the wealth of Western societies. This will be accompanied by massive migration of young workers from other parts of the world to meet the economic needs of many countries. Government will play a major role in allocating services, such as health care and balancing the needs of different segments of society, particularly different age groups. Except for specialized functions, central governments will recede in importance and local governments will become more important. Regional levels of government will become increasingly irrelevant. Small, ubiquitous, wireless technologies will make information and services widely available, but at the expense of personal privacy because a network of sensors will record all kinds of daily activities.

2. Global networked synchronous and ubiquitous mobile government: The essence of this scenario is that technology has made geographic location irrelevant. Citizens will move across borders freely, government will not restrict access and citizens will take part in all decisions that affect them. Government will take on a moderator and gatekeeper role. Biometrics as a form of identification will be commonplace. Smart cards, sensors, mobile technologies of all kinds abound.

3. Local wins: The Me, I, My World of mass selfsegregation: After years of increasing globalization, and a blurring of sense of place, people react by selfselecting into communities of "sameness" using IT to keep them connected to (but still apart from) the rest of the world. In this scenario, everyone has access to technology therefore every person can select the community of interest he/she wishes to associated with. People migrate to these communities with ease and may choose to live in small enclaves composed of people who are most like them in terms of interests, economic status, religion, etc. All their face-to-face interactions take place in these small units, all other interactions, including those with governments take place virtually. Citizens can choose which government they wish to interact with and obtain services from - a person living in one place could choose to pay taxes and take advantage of the services of a different place. Governments thus compete for citizen attention and support.

4. Strong nomadic individuals: Central government will have become a weak except for special functions like security and defense. Government no longer has strong ties to individuals' daily lives. Ubiquitous data stores are accessible by everyone, everywhere. High quality education is widely available through elearning programs devised for you as an individual. Although people have great personal freedom and mobility, they no longer have privacy as sensors are everywhere and personal data is traded outside one's control. Individuals therefore bear a heavy burden to find the information they need and manage the data and technology of their daily lives.

\section{Evaluation of regional workshops}

\subsection{Workshop results}

The scenarios that emerged from the scenario building stage are comprehensive and rich in content and cover the elements of the environment and interaction model. In considering these alternative scenarios, we want to stress that parts of each of these scenarios could play out at different times in different regions or among different groups. So of prime importance is to understand those dimensions resulting in alternative futures, where the idea is that the 'real' future for e-government in 2020 lies in some combination of these wide-ranging possibilities.

In the limited number of pages we are not able to discuss all topics mentioned in the scenarios. A full description can be found in [7] including a compilation of the topics mentioned in each scenario and a count of the number of times a topic occurred. From the scenarios and the topics of interests of each scenario a number of dimensions were derived. The dimensions make up different types of futures and are briefly discussed in the following subsections. We clustered the dimensions into the following three types:

1. Agreed on dimensions. Dimensions were there was general agreement regarding both the nature and direction, e.g. aging population. Ageing will very likely influence the future and there is no doubt that the population.

2. Uncertain dimensions. Dimensions that will likely influence the future, however there is no consensus about the direction, e.g. privacy will likely influence the futures, however in somne scenarios more privacy and in others less privacy was expected;

3. Infrequently mentioned dimensions, dimensions that were part of one, or a small number of, scenarios and are worth mentioning, as they might likely influence the future.

The different categories of dimensions are not mutually exclusive and aspects in one category might influence other categories. Therefore several topics, 
e.g. privacy, participation and so on, are discussed in more than one category.

\subsection{Environmental dimensions category}

5.2.1 Agreed on dimensions, Not surprisingly, in all scenarios ageing, unemployment and the role of immigration were mentioned. Often the role of the individual was mentioned. The individual can belong to global and multiple communities and might become globetrotters. In several scenarios society becomes extremely individual. This was mentioned often together with a divide into a two class society whereby one group of citizens is multi-cultured, well-educated and well-off and the other has less and has no opportunities to improve their position. Consequently e-learning was several times mentioned as an important element. An ageing society requires lifelong learning and retirement at a higher age. 2020 is expected to become a real knowledge economy and we are able to deal with large amounts of information.

5.2.2 Uncertain dimensions, Changing democratic values were mentioned several times, however, how democratic values will be shaped in the future can take several directions. In particular in one scenario government becomes and executes total control. From this it follows a strongly regulated society, which is enforced using control and monitoring technology. The biggest problem will be possibilities for total surveillance and the "black technologies". One extreme outcome is that the separation of powers is repealed and sacrificed to the higher goal of security and control. Values (e.g. privacy protection) are subordinated to the public welfare. In central Europe focus is more on aspects related to monitoring and data misuse than for example in northern Europe. Several times it was mentioned that privacy would disappear and will not be an issue anymore. One time, it was expected that privacy will be better understood and therefore it could be better protected and might depend on the content.

Cybercrime, terrorism, fraud (prevention) and the need for virtual protection were also mentioned in several scenarios. It was even mentioned that government would provide a protected virtual environment for every citizen. Moreover multicultural values can result in frequent clashes and terror, but might also result in a shared culture with a common enemy.

The role of political parties and representation was often mentioned. Political parties are expected to have less influence and the role of more radical parties will increase. Governance would involve more people. Specific issues could be decided on the level of communities of interests. For this purpose governments participate in communities. Often it was expected that the hierarchical system of the society would be flattened. A significant increase in the role of communities and individuals was sometimes expected. On the other hand the rise of a new social system where people would take care of each other was also mentioned.

For the European Union, concern whether it would integrate and become strong or becomes more fragmented or organized at a regional level was addressed several times. Also the degree of cooperation and competition among countries is an important dimension. One time it was mentioned that the EU is expected to extend farther to the east and also was mentioned seamlessly trading with Russia as part of a kind of extended Schengen agreement.

5.2.3 Infrequently mentioned dimensions, In two scenarios, the role of energy facilities and dealing with waste and environmental pollution were expected to impact the e-government future. This might result in local energy production that needs to be controlled by government. Also a larger divide might exist in the world between democratic and non-democratic countries was mentioned once.

\subsection{Interaction dimensions category}

5.3.1 Agreed on dimensions, Universal access to all government services and the possibility for eparticipation from any place at any time was found in all workshops. Voice control was viewed many times as the key interaction technology. One time holographic technologies were mentioned to provide citizens the use of and ability to fully act in virtual space. Hence spatial borders will be overcome and offer physical presence technologies including body contact, smell, and so on. New ways of communication will be possible out of audio-visual communication, (e.g. direct projection). Sometimes this was related to the emergence of new types of borders.

Many times the issue of information overload and processing was mentioned. Media richness will increase, as well as automatic workflows. In order to manage the overload of information and solving the problem of finding the information needed a focus on content management, knowledge management, data mining, information retrieval, artificial intelligence, semantic web technologies and ontology developments would develop. 
5.3.2 Uncertain dimensions, Uncertain is if borders will change and in which direction or current borders will be enforced. Apart from national and EU borders, new horizontal and vertical communities arise having outside borders and interoperability borders, i.e. areas that can seamlessly communicate with each other and areas that cannot. Governments become involved in communities to draw attention to possible services, which would otherwise not be known by community members, and to ensure that community members participate in policy making.

The question of what makes up the core of governments was addressed in all workshops, however the direction varied. Would government shrink or grow? Provide services or focus on policy-making? Sometimes it was expected that governments will act as a coordinator, promoter, or fosterer rather than direct enforcer. Governments might only guarantee democracy, beyond that it might withdraw totally. In other scenarios service provisioning was left to the market and government makes the policies and sets the conditions for effective service provisioning. In other scenarios government provided only services to those who have no access to them, while others could buy them on the market.

The number and level of services provided in the scenarios was often different. In some scenarios government would provides only a few basic services. Infrastructure or security services would no longer be offered and public social security would be limited to a minimum. Alternatively, with ageing society, new types of services and automatic provisioning of services could be expected to be needed.

One of the main topics mentioned was the possibility for automatic data collection and monitoring and possible misuse. In one extreme every person has an implanted chip and is monitored day and night. Monitoring and intervention become so overgrown that a person might even not be able to use a car or public transport and should cycle to their work if their cholesterol level is too high.

Several times it was expected that the need for democratic participation mechanisms will be greater than before and will lead to more power for the individual. Contrary to this other scenarios expected that society will have a larger impact on decision making. Participation would not only be possible through voting but via the exchange of opinions and feedback. Not only would voters participate but all people at all ages, including children, and teenagers would let the government know what they need and want.
5.3.3 Infrequently mentioned dimensions, It was two times expected that governments will be strongly impacted by increasing industry lobbyism.

\subsection{Governmental dimensions category}

5.4.1 Agreed on dimensions, In general it is expected that the society and interaction with government look completely different from today. Governments are expected to be able to react in realtime to protect citizens' interests, prevent cyber fraud and respond to crisis situations that might be caused by such situations as religious conflicts. Protection of Internet activity of citizens might become a prime concern for government.

Services provided through ICTs would become more user-centric. A user will be able to choose services he/she needs and access them in way that is comfortable for him/her. Governmental bodies should only have access to information they need and contentdependent privacy mechanisms are necessary.

5.4.2 Uncertain dimensions, It is uncertain whether EU level decisions and general power might increase or decrease and national level governments' power might decrease. EU-level bureaucracy and transparency could either decrease or increase. Transparency might increase in both directions citizens would be able to watch government's actions and government would be more able to control citizens.

There will be a high integration of governmental and other (private) information systems. The boundaries between public and private systems might be blurred.

The management and adoption of global standards in many fields could become more important. The adaptation of these standards (or the revision to deal with different requirements) by European member states is considered especially uncertain.

In order to enable governments to focus on their essential activities, many public tasks might be privatized and outsourced. A decentralization of customized public service provisioning and a centralization of ICT, procurement and service provisioning were often mentioned to guarantee efficiency. Sometimes it was expected that large data centers would come into existence serving the whole European Union. Which direction is unclear as some expected a power struggle between public agencies and countries to deliver services and the roles of local government might become less or stronger. 
The possibility of shared and mutual understanding among cultures was considered as highly uncertain. Software able to summarize discussions and bridge cultures and languages is expected to emerge. Moreover standardization of requirements for egovernment seems to be necessary, but what should be performed by governments and what delivered by the market?

The decision-making process will change substantially. The partnership between different interest groups will be organized via networks. New types of decision-making frameworks and the proliferation of (business or citizens) choice are expected. More emphasis on managing change and transformation, simulation and animation of policy options is expected to dominate. Transparency and visualizations are expected to become more necessary as the complexity increases with the networked economy. Realizing a transparent and accountability government without corruption was often viewed as an ideal that would be reachable using technology.

There will be a high integration of governmental and public information systems. Semantic networks and interoperability will support widespread ICT usage. Furthermore ICT will help to overcome the differences and problems resulting from different languages.

\subsubsection{Infrequently mentioned dimensions, Once} it was mentioned that the taxation system might be subject to changes, might be virtual, and based on physical consumption or income based. Moreover, there might be only one tax system with local variants.

Politicians and governments would be responsible to guarantee a high degree of transparency, privacy, security and trust. In order to guarantee privacy and trust, as well as security of public services, ICT solutions related to authentication, identification, privacy enhancing methods, data protection, and data security requires further research and development. Some expected that a user orientation would demand more data. Privacy would therefore be given up. Whereas others expected that mechanisms ensuring privacy would appear and at the same time have high levels of customer orientation.

In several workshops technology was viewed as an instrument to solve the problems of society. This view often involved existing technologies and no disruptive technologies enabling breakthroughs were mentioned.

\section{Conclusions}

If we already knew the future, we would not need scenarios depicting possible futures. The scenario approach is a way for policy-makers to describe different futures and to understand different dimensions that make up a possible future. Since the future cannot simply be viewed as a continuation of the past, regional workshops with experts from governments, information and communication technology industry and academia were conducted to stimulate interaction and creativity in order to derive scenarios. Parts of each of the 15 developed scenarios could play out at different times in different regions or among different groups. So it is very important to understand those dimensions resulting in alternative futures where the idea is that the 'real' future for egovernment in 2020 lies in some combination of these wide-ranging possibilities. Nevertheless, in order to develop a robust policy-relevant research agenda, it is important to consider the implications of the full range of future possibilities that is encapsulated by the divergent dimensions.

In the workshops a wide variety of topics and dimensions were identified which lead to alternative futures. In general, in all scenarios technology is not considered as key element of the future. Rather innovations are expected from the use of existing technologies within a context. One cause of this might be that disruptive technology cannot be predicted. On the other hand, most of the participants expected that future e-government challenges would come from the changes in the societal and interaction environments which are more likely to determine the methods of monitoring, interaction, collaboration, policy making and enforcement. As such, the participants expected that societal and modernization of government aspects will primarily influence the different futures. Technology was viewed as an instrument to help solve problems of society.

The general view is that society in 2020 will be different from now and that the current struggle with the translation of these technologies into government applications will be solved. Thinking in terms of cooperation in communities, solving the privacy problems and local focus to stay close to citizens seems to be the vision of most of the session participants. The different governmental institutions must realise that connectivity at a semantic level with other governments, but also with private parties, is of importance and that they all must take responsibility in this toward the information society in general. It is expected that breakthroughs in e-government will not 
occur because of a specific application or disruptive technology, but primarily due to the relation of the government with its citizens.

In our future research, a gap analysis and research roadmap to support policy-makers will be developed. In the gap analysis the weaknesses, problems and needs of future research will be analyzed in order to accomplish wanted futures and to avoid unwanted ones. Based on these results, a sequence of roadmapping workshops will be carried out to develop a research plan for e-government that should pave the way toward the future. The research roadmap should guide strategic bodies to launch appropriate egovernment research programs. Accordingly, the research roadmap is aimed at addressing research challenges and actions to take in technical, organizational, social, economic, and political dimensions. The scenarios help to draw attention to the variety of developments and visions that could be the future. For Europe, this variety should help policymakers to leverage the potential of the diversity and take into account the differences when transforming the European government landscape into a coherent community. On a more global scale, the scenarios offer insights into broad social, political, and demographic concerns that will shape both government and society in the coming decades.

\section{Acknowledgement}

This paper is based on research within the eGovRTD2020 (Roadmapping eGovernment research 2020, IST-2004-4-27139) project. A specific support action co-funded by the European Commission under the 6th framework program of IST with the following partners: University of Koblenz-Landau (coordinator, DE), Delft University of Technology (NL), Center for Technology and Innovation Management (DE), Mykolas Romeris University (LT), University of Maribor (SI), European Institute of Public Administration (NL), Hautes Etudes Commerciales (FR), Australian National University, Center for applied philosophy (AU), Center for Technology in Government, University at Albany-SUNY (USA).

\section{References}

[1] M. Bicking, M. Janssen, and M.A. Wimmer, eGovernment 2020: Towards a Roadmap for future eGovernment research in Europe. EChallenges conference, Barcelona, Spain, 2006.
[2] W.A.G.A. Bouwman and P.A. van der Duin "Technological forecasting and scenarios matter: research into the use of information and communication technology in the home environment in 2010", Foresight, 5, 4, 2003, pp. 8-20.

[3] J.M. Carroll (1995). Scenario-Based Design: Envisioning Work and Technology in System Development, Wiley.

[4] Coates, V., M. Farooque, R. Klavans, K. Lapid, H.A. Linstone, C. Pistorius \& A.L. Porter, "On the future of technological forecasting", Technological Forecasting \& Social Change”, Vol.67, No. 1, 2001, pp.1-17

[5] E. Dammers, Leren van de toekomst. Over de rol van scenario's bij strategische beleidsvorming. Delft: Eburon, 2000.

[6] P. van der Duin, (2006). Qualitative futures research for innovation. Doctoral dissertation, Delft University of Technology, Delft, The Netherlands.

[7] M. Janssen, P. van der Duin, T. Monasso, M. Bicking and M.A. Wimmer, Scenario report (including regional workshop reports). Deliverable D2.1 Roadmapping eGovernment RTD 2020 [http://www.egovrtd2020.org/, last accessed September 2006].

[8] F.E. Emery and E.L. Trist, "The causal texture of organisational environments", Human Relations, vol. 18, 1965, pp. 21-32.

[9] J. Glenn, (ed.) (1999). Futures research methodology. Washington: American Council for the United Nations University [on CD Rom: version 1.0].

[10] K. Van der Heijden, Scenarios: the art of strategic conversation. Chichester: Wiley, 1996.

[11] A. Horton, "Forefront: a simple guide to successful foresight", Foresight, vol. 1, no. 1 (1999), pp.5-9.

[12] G. Johnson, K. Scholes and Richard Whittingon, Exploring Corporate Strategy (Prentice Hall, 2002).

[13] H. Kubicek and H. Westholm "Scenarios for future use of e-democracy tools in Europe. International Journal of Electronic Government Research, vol. 1, no. 3, (2005) pp. 33-50.

[14] B.R. Martin, "Foresight in Science and Technology", Technology Analysis \& Strategic Management, vol. 7, No. 2, (1995), pp.139-168.

[15] May, G. (1996). The future is ours. London: Adamantine Press.

[16] P.W.F. van Notten, J. Rotmans, M.B.A. van Asselt and Dale S. Rothman, "An updated scenario typology", Futures, Vol. 35, No. 5 (2003), pp.423-443.

[17] Schwartz, P. (1991). The art of the long view. Chichester: John Wiley \& Sons.

[18] M.A. Wimmer, "Integrated service modeling for online one-stop Government", EM - Electronic Markets, special issue on e-Government, Vol. 12, No. 3, (2002) pp. 1-8. 